\title{
Indissociabilidade entre o ensino, pesquisa e extensão no ensino superior
}

\author{
The straight connection among teaching, research and extension at higher education \\ Indissociabilidad entre la enseñanza, la investigación y la extensión en la enseñanza \\ superior
}

Paulo Ricardo Dias de Sousa ${ }^{1 *}$, lara Rege Lima Sousa ${ }^{2}$, Bruna Furtado Sena de Queiroz ${ }^{3}$, Kátia Lima Braga ${ }^{4}$, Marielle Cipriano de Moura ${ }^{4}$, Francisca Jessica de Sousa Oliveira ${ }^{1}$, Annielson de Souza Costa 5 .

\section{RESUMO}

Objetivos: Descrever a implementação do princípio da indissociabilidade entre ensino, pesquisa e extensão no ensino superior. Métodos: Trata-se de estudo de revisão integrativa de literatura. A seleção do material bibliográfico para este estudo ocorreu nos meses de janeiro a março de 2019, na base de dados da Biblioteca Virtual em Saúde (BVS), que incluem as bases, LILACS - BIREME (Bases de dados da literatura Latino Americana, em Ciências de Saúde) e Scielo - Scientific Electronic Library Online. Os descritores foram encontrados no Descritores em Ciências da Saúde (DeCS), Ensino superior, Extensão comunitária e Pesquisa. Resultados: Os estudos analisados reconhecem que a função do princípio consiste em embasar o ensino universitário, a partir da integração dos três pilares do ensino superior, de modo a formar profissionais cada vez mais capacitados, aliando a teoria com a prática, o conhecimento científico e empírico. O fazer universitário a partir desse pressuposto implica no reconhecimento de que a formação do professor deve ocorrer articulada à vivência do real, fundada em uma relação entre teoria e prática. Conclusão: No entanto, os estudos revelam que essa integração não ocorre de maneira efetiva, com o predomínio do ensino nas universidades, sem articulação com a pesquisa e a extensão.

Palavras-chave: Ensino superior, Extensão comunitária, Pesquisa.

\begin{abstract}
Objectives: To describe the implementation of the principle of inseparability between teaching, research and extension in higher education. Methods: This is an integrative literature review study. The selection of bibliographic material for this study took place from January to March 2019, in the Virtual Health Library (VHL) database, which includes the databases LILACS - BIREME (Latin American Science Databases). Health) and Scielo - Scientific Electronic Library Online. The descriptors were found in Descriptors in Health Sciences (DeCS), Higher Education, Community Extension and Research. Results: The studies analyzed recognize that the principle's function is to base university education, based on the integration of the three pillars of higher education, in order to train increasingly qualified professionals, combining theory with practice, scientific knowledge and empirical. University practice based on this assumption implies the recognition that teacher education must occur in conjunction with the experience of reality, based on a relationship between theory and practice. Conclusion: However, studies show that this integration does not occur effectively, with the
\end{abstract}

${ }^{1}$ Centro universitário Santo Agostinho (UNIFSA), Teresina- Piauí. *E-mail: paulo-ricardodias@outlook.com ${ }^{2}$ Centro Universitário UINOVAFAPI (UNINOVAFAPI), Teresina- Piauí.

${ }^{3}$ Faculdade Integral Diferencial - FACID-WYDEN, Teresina- Piauí.

${ }^{4}$ Universidade Estadual do Maranhão -UEMA, Timon- Maranhão

${ }^{5}$ Faculdade de Medicina FMUSP, Universidade de São Paulo, São Paulo.

SUBMETIDO EM: 5/2019

ACEITO EM: 6/2019

PUBLICADO EM: 9/2019

REAS/EJCH | Vol.Sup.32 | e938 | DOI: https://doi.org/10.25248/reas.e938.2019 Página 1 de 6 
predominance of teaching in universities, without articulation with research and extension. Keywords: Higher Education, Community Extension, Research.

\section{RESUMEN}

Objetivos: Describir la implementación del principio de inseparabilidad entre la enseñanza, la investigación y la extensión en la educación superior. Métodos: Este es un estudio de revisión de literatura integradora. La selección de material bibliográfico para este estudio se realizó de enero a marzo de 2019, en la base de datos de la Biblioteca Virtual en Salud (BVS), que incluye las bases de datos LILACS - BIREME (Bases de datos científicas latinoamericanas). Salud) y Scielo - Scientific Electronic Library Online. Los descriptores se encontraron en Descriptores en Ciencias de la Salud (DeCS), Educación Superior, Extensión Comunitaria e Investigación. Resultados: los estudios analizados reconocen que la función del principio es basar la educación universitaria, basada en la integración de los tres pilares de la educación superior, para formar profesionales cada vez más calificados, combinando teoría con práctica, conocimiento científico y empírico La práctica universitaria basada en este supuesto implica el reconocimiento de que la formación del profesorado debe realizarse en conjunción con la experiencia de la realidad, basada en una relación entre la teoría y la práctica. Conclusión: Sin embargo, los estudios muestran que esta integración no se produce eficazmente, con el predominio de la enseñanza en las universidades, sin articulación con la investigación y la extensión.

Palabras clave: Educación superior, Extensión comunitaria, Investigación.

\section{INTRODUÇÃO}

Devido às mudanças, ao longo dos anos, na maneira e na velocidade como o homem absorve e transmite conhecimento, seria insustentável manter uma metodologia de aprendizado tradicional, em que os alunos se mantinham na posição de ouvintes inertes e um professor apresentava conceitos e diretrizes rígidos de um tópico em específico (BAROTO A et al., 2018).

A formação profissional, desde a graduação universitária, consiste em um grande desafio do século XXI em vários países, sobretudo relacionado aos profissionais da área da saúde. Processos educativos devem possibilitar a inserção dos estudantes nos serviços com o objetivo de aprender com situações concretas de trabalho e atuem com maior resolubilidade e efetividade a partir de experiências educativas baseadas em competências globais e locais para o cuidado em saúde (PINA-OLIVEIRA AA et al., 2014).

Neste contexto, com o fomento as modificações nas metodologias de ensino superior, a Constituição Brasileira de 1988 estabelece mediante o artigo 207 a indissociabilidade entre pesquisa, ensino e extensão como princípio constitucional, impreterível para o processo de formação na Universidade brasileira, não podendo ser fragmentado (BRASIL, 1988).

Portanto, o princípio não pode ser separado ou pautado de forma isolada na Universidade, pois consiste em um instrumento teórico de direcionamento para o fortalecimento do ensino público no Brasil, contemplando ações no âmbito da universidade e da comunidade, considerando a corresponsabilidade entre os gestores, profissionais e comunidade em geral (GONÇALVES NG, 2015).

O Fórum Nacional de Pró-Reitores de Extensão das Instituições de Ensino Superior Públicas Brasileiras (FORPROEX) reitera que são também indissociáveis: Interação Dialógica Interdisciplinaridade e Inter profissionalidade, Indivisibilidade entre Ensino-Pesquisa-Extensão, Impacto na Formação do Estudante e Impacto e Transformação Social compartilham desafios que decorrem da história e da conjuntura atual sobre a Universidade pública (GONÇALVES NG, 2015).

Desta forma, modernamente, a construção do saber induz que o conhecimento seja não apenas sistematizado e gerenciado por um professor, mas constantemente estimulado e reelaborado, promovendo novos espaços de práticas e de reflexão. Para tanto, acredita-se na formação profissional mediante um processo de ensino e aprendizagem de habilidades e competências técnicas; de competências científicas 
com disponibilização de métodos e conteúdo de conhecimento; e de competência cidadã, pelo estímulo do estudante para a tomada de consciência de sua existência histórica e social (BORATO A et al., 2018).

Deve-se reconhecer a necessidade de os professores estarem capacitados para assumirem uma nova pedagogia que permita ser efetiva a tridimensionalidade do fazer universitário. Contudo, é evidente a necessidade de analisar a integração dos pilares da universidade conforme a literatura, pois os mesmos norteiam as ações para formação no ensino superior a partir da democratização do ensino nas universidades. Desde modo este estudo tem como objetivo descrever a implementação do princípio da indissociabilidade entre ensino, pesquisa e extensão no ensino superior.

\section{MÉTODOS}

Trata-se de uma revisão integrativa de literatura que é um método de pesquisa utilizado desde 1980, época em que surgiu a Prática Baseada em Evidências (PBE), que envolve a sistematização e publicação dos resultados de uma pesquisa bibliográfica em saúde, para que possam ser úteis na assistência à saúde, acentuando a importância da pesquisa acadêmica atrelada à prática clínica com a finalidade de realizar a integração entre estas duas(MENDES KDS et al., 2008).

Este método de pesquisa permite a síntese de múltiplos estudos publicados e possibilita conclusões gerais a respeito de uma particular área de estudo. É um método valioso para a enfermagem, pois muitas vezes os profissionais não têm tempo para realizar a leitura de todo o conhecimento científico disponível devido ao volume alto, além da dificuldade para realizar a análise crítica dos estudos (MENDES KDS et al., 2008).

Mendes KDS, Silveira CCP e Galvão CM (2008) ressaltam ainda, que a pesquisa integrativa segue os mesmos estágios da pesquisa convencional, dividindo-se nas seguintes etapas: identificação do tema e elaboração das questões norteadoras para a realização da pesquisa; estabelecimento dos critérios de inclusão e de exclusão; definição das informações a serem extraídas e sua categorização; analise dos estudos incluídos na revisão integrativa; interpretação dos resultados e por fim, a apresentação da revisão e síntese do conhecimento.

A seleção do material bibliográfico para este estudo ocorreu nos meses de janeiro a março de 2019, na base de dados da Biblioteca Virtual em Saúde (BVS), que incluem as bases, LILACS - BIREME (Bases de dados da literatura Latino Americana, em Ciências de Saúde) e Scielo - Scientific Electronic Library Online.

Os descritores foram encontrados no Descritor em Ciências da Saúde (DeCS) e se constituem os seguintes: Ensino superior, Extensão comunitária e Pesquisa.

Para os critérios de inclusão das amostras selecionaram-se artigos completos publicados no período de 2007 a 2017, em periódicos nacionais, que estivessem disponíveis em língua portuguesa, em texto completo, e que contemplem a temática abordada.

Os critérios de exclusão se configuram em artigos que não contemplem o período de publicação ou que fuja do tema e artigos não completos.

Aplicando-se os critérios de inclusão e exclusão restaram 23 artigos, dos quais 9 se enquadram com os objetivos do estudo, assim, foram analisados e discutidos. Com base neste contexto, foram analisados e discutidos.

\section{RESULTADOS}

Apresenta- se a seguir o quadro com as características dos 9 artigos estudados, descrevendo segundo a ordem dos artigos, os autores e ano de publicação, títulos e objetivo (Quadro 1).

Com base no agrupamento e organização dos artigos estudados, expostos no quadro acima e análise textual dos mesmos, deram origem as categorias que foram discutidas a luz do referencial teórico sobre 0 
tema, denominado: "A Indissociabilidade: princípio constitucional e os três pilares do ensino superior: ensino, pesquisa e extensão".

Quadro 1 - Distribuição das características das produções analisadas, Teresina, Piauí, 2019.

\begin{tabular}{|c|c|c|c|}
\hline $\begin{array}{l}\text { Ordem dos } \\
\text { artigos }\end{array}$ & Autor & Título & Objetivo \\
\hline 1 & $\begin{array}{ll}\text { Cunha } & \text { Ml } \\
(2011) & \end{array}$ & $\begin{array}{l}\text { Indissociabilidade entre ensino } \\
\text { e pesquisa: a qualidade da } \\
\text { graduação em tempos de } \\
\text { democratização }\end{array}$ & $\begin{array}{l}\text { Compreender as formas de produção } \\
\text { desse sobre a indissociabilidade e as } \\
\text { recontextualizações necessárias para } \\
\text { inserir-se na atualidade }\end{array}$ \\
\hline 2 & $\begin{array}{l}\text { Chesani FH, } \\
\text { et al (2017) }\end{array}$ & $\begin{array}{llcrr}\text { A indissociabilidade } & \text { entre } & \text { a } \\
\text { extensão, o ensino } & \text { e } & \text { a } \\
\text { pesquisa: } 0 & \text { tripé } & & \text { da } \\
\text { universidade } & & & & \end{array}$ & $\begin{array}{l}\text { Apresentar como foi estimulado o } \\
\text { processo de um projeto de extensão } \\
\text { que preconiza a indissociabilidade entre } \\
\text { o ensino, a pesquisa e a extensão }\end{array}$ \\
\hline 3 & $\begin{array}{l}\text { Gonçalves } \\
\text { NG, et al } \\
(2014)\end{array}$ & $\begin{array}{l}\text { A extenção na universidade } \\
\text { federal do paraná: constituição } \\
\text { histórica }\end{array}$ & $\begin{array}{l}\text { Analisar as concepções de extensão } \\
\text { que permearam e permeiam a história } \\
\text { da Universidade Federal do Paraná }\end{array}$ \\
\hline 4 & $\begin{array}{l}\text { Gonçalves } \\
\text { NG (2015) }\end{array}$ & $\begin{array}{l}\text { Indissociabilidade entre ensino, } \\
\text { pesquisa e extensão: um } \\
\text { princípio necessário }\end{array}$ & $\begin{array}{l}\text { Discutir, de forma contextualizada, o } \\
\text { princípio da indissociabilidade entre } \\
\text { ensino, pesquisa e extensão, no âmbito } \\
\text { universitário no Brasil. }\end{array}$ \\
\hline 5 & $\begin{array}{l}\text { Moita FM, } \\
\text { Andrade FCB } \\
(2009)\end{array}$ & $\begin{array}{l}\text { Ensino-pesquisa-extensão: um } \\
\text { exercício de indissociabilidade } \\
\text { na pós-graduação }\end{array}$ & $\begin{array}{l}\text { Mostrar a importância do princípio da } \\
\text { indissociabilidade entre ensino, } \\
\text { pesquisa e extensão }\end{array}$ \\
\hline 6 & $\begin{array}{l}\text { Puhl } \\
\text { (2016) }\end{array}$ & $\begin{array}{l}\text { O conhecimento e o princípio da } \\
\text { indissociabilidade entre ensino, } \\
\text { pesquisa e extensão }\end{array}$ & $\begin{array}{l}\text { Debater o princípio da } \\
\text { indissociabilidade com vistas a } \\
\text { contribuir para uma compreensão } \\
\text { integrada destas dimensões do fazer } \\
\text { acadêmico, de forma articulada entre si. }\end{array}$ \\
\hline 7 & $\begin{array}{l}\text { Prates EAR, } \\
\text { et al (2017) }\end{array}$ & $\begin{array}{l}\text { Ensino, pesquisa e extensão: } \\
\text { indissociáveis? }\end{array}$ & $\begin{array}{l}\text { Verificar se a indissociabilidade entre } \\
\text { ensino, pesquisa e extensão já se } \\
\text { consolidou no ensino superior }\end{array}$ \\
\hline 8 & $\begin{array}{l}\text { Rosário CL et } \\
\text { al (2013) }\end{array}$ & $\begin{array}{l}\text { Indissociabilidade entre ensino, } \\
\text { pesquisa e extensão } \\
\text { universitária: experiências nos } \\
\text { cursos de licenciatura do } \\
\text { instituto federal de Roraima }\end{array}$ & $\begin{array}{l}\text { Conhecer a visão dos professores sobre } \\
\text { as ações de extensão como articuladora } \\
\text { do ensino e da pesquisa. }\end{array}$ \\
\hline 9 & $\begin{array}{l}\text { Tavares } \\
\text { DMS et al } \\
(2007)\end{array}$ & 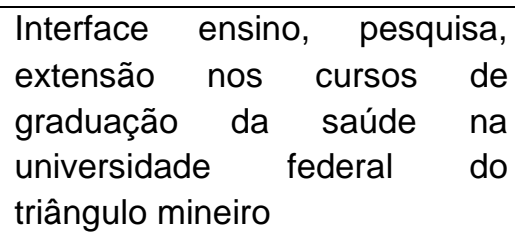 & $\begin{array}{l}\text { Descrever e avaliar o programa de } \\
\text { extensão "Integrando saberes para a } \\
\text { atenção integral à família" }\end{array}$ \\
\hline
\end{tabular}

Fonte: Sousa PRD, Sousa IRL, Queiroz BFS, et al, 2019.

\section{DISCUSSÃO}

\section{A Indissociabilidade: princípio constitucional}

Segundo Cunha MI (2011) no Brasil ao longo da história, prevaleceu a compreensão de que a relação de indissociabilidade do ensino, pesquisa e extensão é que caracteriza a qualidade da educação superior e que a compreensão de conhecimento sustenta essa relação, sempre a entendendo num contexto cultural. 
Para Puhl MJ (2016) o princípio tem como função direcionar as ações para o alcance das metas e objetivos previamente estabelecidos, considerando as etapas a serem seguidas. É um instrumento fundamental para o planejamento da educação no ensino superior, bem como, para superação do modelo tecnocrata de ensino.

O autor supracitado ressalta a função do princípio de embasar o ensino universitário de maneira pedagógica, de modo a formar profissionais cada vez mais capacitados, aliando a teoria com a prática, o conhecimento científico e empírico. Moita FM e Andrade FCB (2009) afirmam que ao empregar a articulação ensino, pesquisa e extensão na universidade é fundamental levar em consideração a integração do saber cientifico com o conhecimento popular, baseado na cultura vivenciada dentro da comunidade.

Para Rosário CL, et al (2013) as ações da universidade a partir dessa premissa, devem enfocar na formação do professor, com a vivência na comunidade, através da extensão, contribuindo para o fomento de pesquisas, por meio de uma diagnostico situacional da prática, proporcionando assim, um aprofundamento do conhecimento apreendido na sala de aula.

A indissociabilidade entre ensino, pesquisa e extensão impulsiona os docentes e os discentes universitários à articulação de saberes da experiência, o saber do conhecimento e o saber pedagógico. Essa integração permite a adoção de práticas de ensino prazerosas, crítica e reflexiva, superando os métodos tradicionais (TAVARES DMS et al., 2007).

\section{Os três pilares do ensino superior: ensino, pesquisa e extensão.}

A universidade assume seu papel na formação de profissionais quando integra o ensino, pesquisa e extensão, tornando o conhecimento mais acessível à população, possibilitando assim, uma a troca integrada entre o saber popular adquirido de forma cultural e o cientifico através da universidade.

Entretanto, Moita FM e Andrade FCB (2009) ressaltam o predomínio do ensino e da pesquisa na maioria das instituições de ensino superior, negligenciando a extensão universitária. Para os autores, à extensão, em particular, a indissociabilidade oferece à formação discente um espaço capaz de "permitir a atualização dos conteúdos programáticos e simultaneamente embasar os novos profissionais numa ética de cidadania". Pesquisa, ensino e extensão articulam-se, na formação acadêmica, de modo a promover uma consciência profissional eticamente fundamentada e empiricamente atualizada.

Chesani FH, et al (2017) salienta que não há relevância social da pesquisa sem a indissociabilidade. Neste sentido, o ensino e a extensão não são complementos da pesquisa, pois devem estar integradas entre si de forma contínua, embasando-a no aprofundamento de questões cientificas e populares relevantes.

Para Gonçalves NG (2015) a extensão assumida como uma das funções da Universidade, de certa forma, permite que as ações de Ensino, de Pesquisa e de Extensão permaneçam dissociadas, ou seja, a existência do ensino, pesquisa e extensão nas universidades não significa necessariamente que estes estejam sendo indissociáveis ou integrados. Assim, a extensão avança parcialmente em sua conquista por espaço e legitimidade interna, e o Ensino e a Pesquisa não sofrem necessariamente mudanças.

A extensão realizada de maneira isolada, como atividade complementar, através de cursos, projetos, eventos e programas, não implica como parte do princípio constitucional de indissociabilidade, mas como uma atividade formal, inflexível e padronizada, que uma vez, não integrada ao ensino e pesquisa cursa com pouca efetividade na absorção de conhecimento e, por conseguinte na formação profissional (ROSÁRIA CL, et al., 2016)

De acordo com Puhl MJ (2016) a integração entre pesquisa, ensino e extensão contribui para superar a dicotomia entre o saber científico e empírico, entre a teoria e prática. $O$ autor destaca a teoria do filosofo kant para explicar a relação entre a indissociabilidade entre os três pilares da educação superior e a produção do conhecimento.

É necessário compreender como os processos de ensinar e aprender são atingidos pela implementação da indissociabilidade entre ensino, pesquisa e extensão. Cunha MI (2011) salienta a mudança da docência centrada no professor para a docência centrada no estudante, através da associação exclusiva entre ensino 
e pesquisa. Todavia, a extensão faz-se indissociável com o ensino e pesquisa, pois promove a interação do estudante com a comunidade e serviços, gerando estímulos para pesquisa.

\section{CONCLUSÃO}

O princípio constitucional que abarca a inerência entre ensino, pesquisa e extensão, uma vez implementado, favorece a troca do conhecimento acadêmico com o popular, por intermédio da extensão comunitária, onde o aluno coloca em pratica os ensinamentos da sala de aula o que contribui para 0 aprofundamento dos conhecimentos, através da pesquisa. No entanto, os estudos revelam que essa integração não ocorre de maneira efetiva, com o predomínio do ensino nas universidades e a extensão quando não é negligenciada é realizada de maneira isolada. Contudo, observa-se a necessidade de estudos, sobretudo na área da saúde, relacionados à efetivação da integração dos três pilares fundamentais para formação acadêmica no ensino superior, de modo a impulsionar sua implementação no ensino universitário.

\section{REFERÊNCIAS}

1. BORATO A, et al. Valoração das práticas de ensino, pesquisa e extensão entre concluintes de Odontologia. Revista da ABENO. 18(1): 103-115, 2018.

2. BRASIL. Constituição da República Federativa do Brasil de 1988. Brasília, 5 out

3. 1988. Disponível em: <http://www.planalto.gov.br/ccivil_03/constituicao/constituicao.htm>. Acesso em:10 fev. 2019

4. CHESANI FH, et al. A indissociabilidade entre a extensão, o ensino e a pesquisa: o tripé da universidade. Rev. Conexão UEPG. Ponta Grossa, v. 13 n.3 - set./dez. 2017

5. CUNHA MI. Indissociabilidade entre ensino e pesquisa: a qualidade da graduação em tempos de democratização. Perspectiva, v. 29, n. 2, p. 443-462, 27 jun. 2011.

6. GONÇALVES NG. Indissociabilidade entre Ensino, Pesquisa e Extensão: um princípio necessário. Rev. PERSPECTIVA, Florianópolis, v. 33, n. 3, p. 1229 - 1256, set./dez. 2015.

7. GONÇALVES NG, et al. Extensão na Universidade Federal do Paraná: constituição histórica. Rev. Extensão em Foco, Curitiba: Editora da UFPR, n.9, p.3-49, jan/jun 2014.

8. MENDES KDS, et al. Revisão integrativa: método de pesquisa para a incorporação de evidências na saúde e na enfermagem. Texto Contexto Enferm, Florianópolis; 17(4): 758-64. 2008.

9. MOITA FM, ANDRADE FCB. Ensino-pesquisa-extensão: um exercício de indissociabilidade na pós-graduação. Rev. Brasileira de Educação. v. 14 n. 41 maio/ago. 2009.

10. PINA-OLIVEIRA AA, et al. Avaliação da Indissociabilidade entre Ensino, Pesquisa e Extensão Relacionada à Promoção do Desenvolvimento Infantil em Instituições de Ensino Superior do Interior Paulista. Revista de Cultura e Extensão USP, v. 12, p. 47-65, 31 out. 2014.

11. PRATES EAR, et al. Ensino, pesquisa e extensão: indissociáveis? Rev. Digital. Buenos Aires, 2017.

12. PUHL MJ. O conhecimento e o princípio da indissociabilidade entre ensino, pesquisa e extensão. Rev. HISTEDBR On-line, Campinas, no 69, p. 222-232, set2016.

13. ROSÁRIO CL, et al. Indissociabilidade entre ensino, pesquisa e extensão universitária: experiências nos cursos de licenciatura do Instituto Federal de Roraima. Gestão e tecnologia para competitividade. out. 2013.

14. TAVARES DMS, et al. Interface ensino, pesquisa, extensão nos cursos de graduação da saúde na universidade federal do triângulo mineiro. Rev Latino-am Enfermagem. v. 15, n. 6, 2007. 